\title{
Label-free biomedical imaging of hydrodynamics in single human cells
}

\author{
Hisanori Fukunaga ${ }^{1}$, Hirofumi Yoshimura ${ }^{2}$, Yukio Nishina ${ }^{3}$, Yoji Nagashima ${ }^{4}$, and Masaru Tachibana ${ }^{2}$ \\ ${ }^{1}$ Yokohama City University School of Medicine, Yokohama, Japan; ${ }^{2}$ Department of Nanosystem Science and ${ }^{3}$ Department of Genome \\ System Science, Graduate School of Nanobioscience, Yokohama City University, Yokohama, Japan; and ${ }^{4}$ Department of Molecular \\ Pathology, Graduate School of Medicine, Yokohama City University, Yokohama, Japan
}

(Received 10 March 2010; and accepted 22 March 2010)

\begin{abstract}
Intracellular hydrodynamics is of considerable importance to regulate cellular functions. Using Raman microspectroscopy and imaging, we visualized the time-dependent changes of water concentration in single human acute promyelocytic leukemia HL-60 cells, and also showed the labelfree biomedical imaging of water molecules, lipids and proteins for analyzing molecular distribution. Taken together, our results demonstrated that we can distinguish between intracellular water and other major cell components, and that when the physiological status of cells changes with time, the Raman spectral intensities for intracellular water concentration also changes. Therefore, this biospectroscopic analysis of intracellular hydrodynamics can provide biomarkers to examine activities in dynamic living systems, and has significant potential in determining cell specificity for novel therapeutic approaches.
\end{abstract}

Intracellular water is not only the principal solvent, but also the critical reactant for the structures and functions of biological macromolecules. Because water provides the dynamic matrix in which all biochemical and biophysical processes occur, intracellular hydrodynamics are of importance in regulating cell functions $(2,3)$. It has been clinically reported that abnormally high water content is generally present in cancer cells, and that the degree of malignancy increases with the extent of cell hydration (6). The development of cell-specific targeting system is of considerable significance for novel therapeutic approaches, and given the fundamental participation of intracellular water content with cell specificity, the investigation of intracellular hydrodynamics has potential in medicine. More knowledge of intracellular hydrodynamics is highly desirable for both biomedical research and clinical practice.

Raman spectroscopy is a suitable method to

Address correspondence to: Hisanori Fukunaga

Yokohama City University School of Medicine, 3-9

Fukuura, Kanazawa-ku, Yokohama 236-0004, Japan

Tel: +81-45-787-2586, Fax: +81-45-786-0191

E-mail: e083070b@yokohama-cu.ac.jp address this issue, because it enables the analysis of molecular characteristics of biological compositions in the cell via the vibrational frequencies of chemical bonds. The greatest benefits of Raman microspectroscopy are its high sensitivity and capacity for non-invasive sensing of molecular species in single living cells $(10,11)$. In addition, Raman imaging can also provide detailed information regarding spatial molecular distributions $(4,7)$. Intracellular water molecules can be sensitively and non-invasively detected using Raman spectroscopic applications. In contrast to nuclear magnetic resonance (NMR) or fluorescence techniques, intracellular water domains can be clearly separated from the extracellular environment, and regions of severely restricted water mobility may be readily identified within the cell (9). Thus, we initiated a practical combination of confocal Raman microspectroscopy and imaging to study intracellular hydrodynamics for future use in medicine.

\section{MATERIALS AND METHODS}

Sample preparation. We performed Raman spectroscopic analysis of human acute promyelocytic leu- 
kemia HL-60 cells as a model in this preliminary study, because the HL-60 cultured cell line is a representative source of human cancer cells for the pharmacotherapeutic study of cell specificity. In addition, it was easy to analyze HL-60 cells with our experimental system, provided they are generally round or ovoid and grow in a single-cell suspension without any tendency to clump or adhere to plastic or glass surface (5). HL-60 cells were incubated at $37^{\circ} \mathrm{C}$ under $5 \% \mathrm{CO}_{2}$ in RPMI 1640 medium (Nissui Pharmaceutical Co, Ltd, Tokyo, Japan) supplemented with $10 \%$ fetal calf serum (Biowest, Nuaillé, France). For sample preparation, HL-60 cells were suspended in $\mathrm{Ca}^{2+}$ - and $\mathrm{Mg}^{2+}$-free phosphatebuffered saline (PBS), and then were placed on a glass slide and cover-slipped. The edges of the cover slip were sealed to prevent evaporation.

Confocal Raman microspectroscopy. Fig. 1 shows the optical apparatus of the Raman microspectroscopy system used. Raman spectra were measured using a confocal Raman microspectrometer (NRS-1000; JASCO, Tokyo, Japan) at $532 \mathrm{~nm}$. The spatial resolution was $1 \mu \mathrm{m}$ in the lateral direction and $2 \mu \mathrm{m}$ in the depth direction. At approximately $1 \mathrm{~mW}$, the laser was focused on a selected cell and the exposure time was $3 \mathrm{~s}$ as non-invasive. The scattered light was collected in a backscattering configuration. All experiments were performed at room temperature.

Label-free biomedical imaging. Label-free biomedical imaging measurements were performed using a specially designed confocal Raman imaging apparatus (Nissan ARC, Japan). The spatial resolution was $400 \mathrm{~nm}$ in both the lateral and vertical directions. A 532-nm laser was focused and its power was approximately $0.5 \mathrm{~mW}$. A confocal Raman image $(20 \mu \mathrm{m} \times 20 \mu \mathrm{m})$ consisted of $5625(75 \times 75)$ pixels with $100 \mu$ s measurements, and it took approximately $10 \mathrm{~min}$ to complete the imaging of a whole cell.

\section{RESULTS AND DISCUSSION}

A three-dimensional dynamic hydrogen bond network of water molecules was responsible for the central component of Raman spectra, and the characteristic broad bands in the $3200-3400 \mathrm{~cm}^{-1}$ region were present (Fig. 2A). This band corresponded to the complex vibrations of $\mathrm{OH}$ and hydrogen bonds of water molecules (8). There is a very close association between an analyte concentration and the Raman scattered light intensity of the analyte. Hence, a plot of analyte band intensity versus ana-

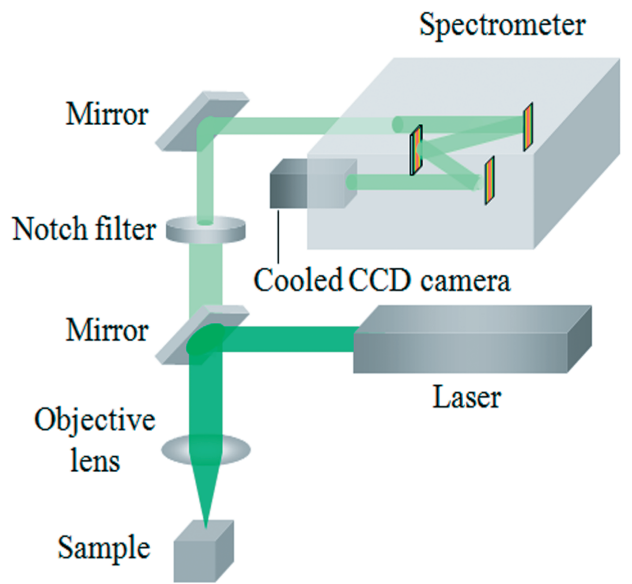

Fig. 1 Optical apparatus of the Raman microspectroscopy system (NRS-1000; JASCO, Tokyo, Japan).

lyte concentration produces a linear curve, demonstrating the correlation between Raman intensity and analyte concentration. Taken together, the water concentration in an individual cell can be sensitively evaluated by intracellular/extracellular Raman intensity ratio in the $3200-3400 \mathrm{~cm}^{-1}$ region.

We detected time-dependent changes in intracellular water concentration and cellular pathophysiological condition using Raman microspectroscopy. The water concentration in a living cell is strictly regulated within a range that is compatible for maintaining the highly organized molecular intracellular network (i.e., intracellular water homeostasis), however, in Fig. 2B the intracellular water concentration gradually increased during this measurement. As shown in Fig. 2C, the cell showed membrane blebbing at $15 \mathrm{~min}$ and then it could not be regarded as alive beyond $30 \mathrm{~min}$. These morphological observations indicated that cellular pathophysiological condition went bad with time. This was likely because the cells were suspended in $\mathrm{Ca}^{2+}$ - and $\mathrm{Mg}^{2+}$-free PBS without oxygen and nutrients, which is an isotonic environment not conducive to cellular metabolic functions. The extracellular environment leads to abnormality or loss of cellular functions and then cell death is induced by disordered essential cell metabolism. Taken together, these results suggested an association between intracellular water concentration and cellular pathophysiological condition. Furthermore, we essentially obtained the same result by analyzing 79 cells, which shows that intracellular water concentration sharply reflects cellular metabolic functions. Thus, we concluded Raman spectral intensity of intracellular water concentration can sensitively indicate cellular condition. 

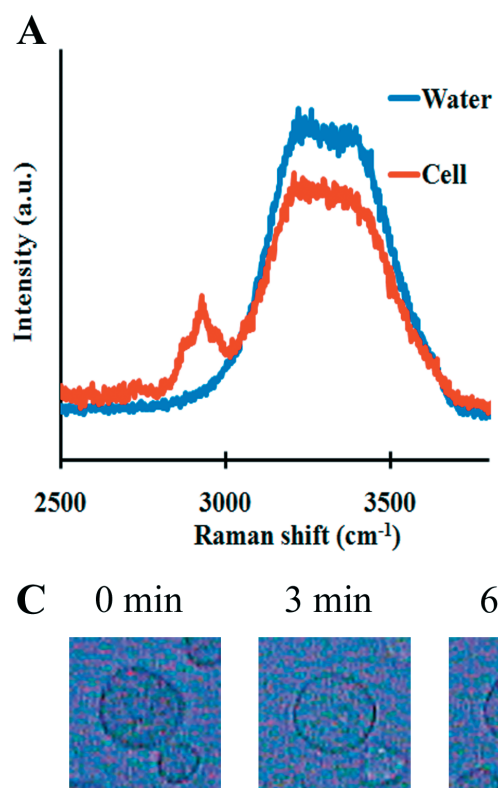

$18 \mathrm{~min}$

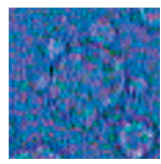

$3 \mathrm{~min}$

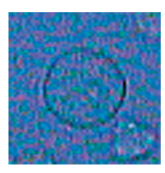

$21 \mathrm{~min}$

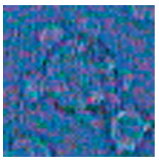

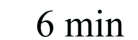

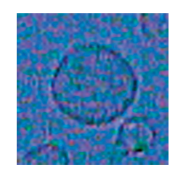

$24 \mathrm{~min}$

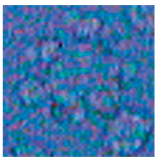

B
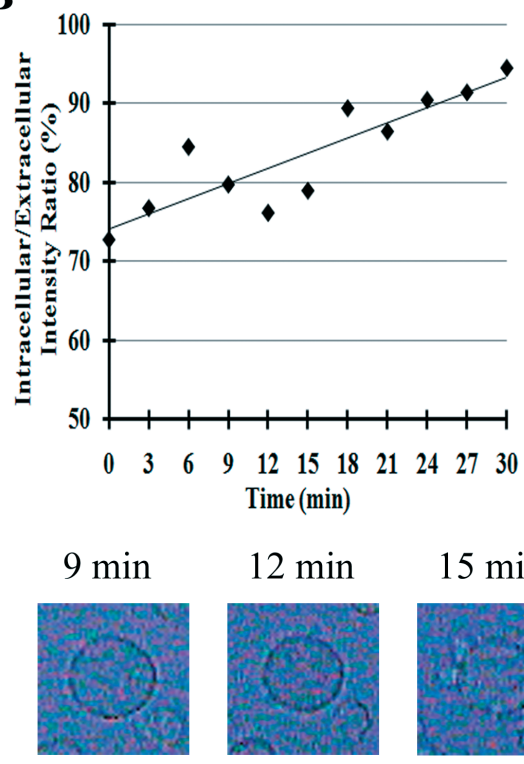

$27 \mathrm{~min}$

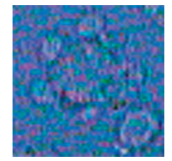

12 min

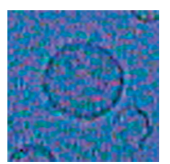

$30 \mathrm{~min}$

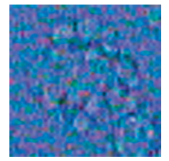

Fig. 2 Time-dependent changes in the water concentration in a HL-60 cell. (A) Water band in the Raman spectrum. The curves indicate the intensities of Raman scattered lights for the cell (red) and water (blue). (B) The time-dependent changes in the intracellular/extracellular intensity ratio. It reflects the water concentration within the cell. (C) The corresponding optical microscopic images. At $15 \mathrm{~min}$, the cell showed membrane blebbing. Scale bar $=10 \mu \mathrm{m}$.

A

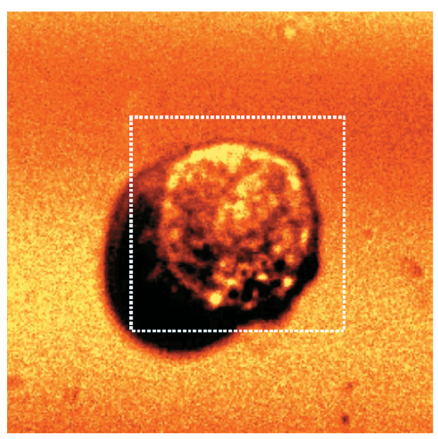

B
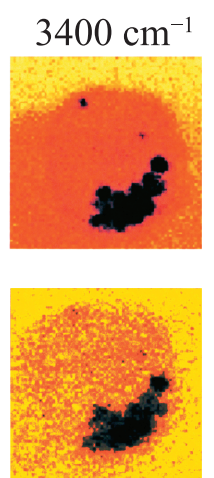
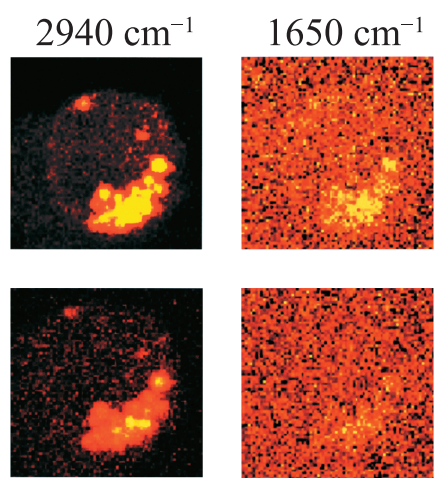

$15 \min$
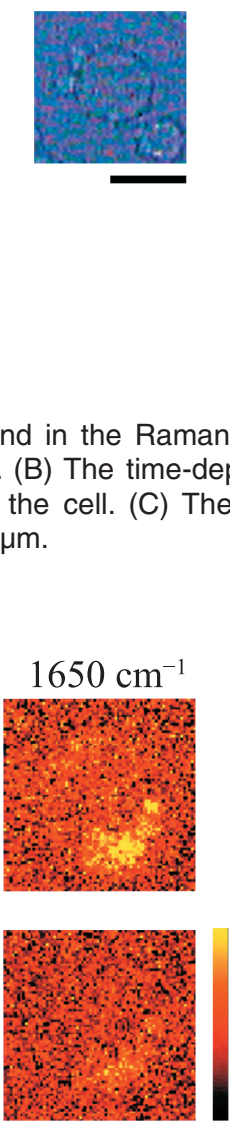

Fig. 3 Label-free biomedical imaging of a HL-60 cell. (A) Confocal laser microscope image. The square area $(20 \times 20 \mu \mathrm{m})$ is measured using confocal Raman imaging. (B) Label-free biomedical images of the HL-60 cell at low (above) and high (below) water concentrations. The imaging patterns of three Raman bands are shown: $3400 \mathrm{~cm}^{-1}$ (water), $2940 \mathrm{~cm}^{-1}$ (lipids) and $1650 \mathrm{~cm}^{-1}$ (proteins). Scale bar $=10 \mu \mathrm{m}$.

We investigated the molecular mechanism of the correlation between intracellular aquatic environment and cell functioning by confocal Raman imaging. In Fig. 3A, the focused laser spot scanned an area that included a selected HL-60 cell. Raman scattered light intensities collected at each point were combined to construct high-contrast maps of the intensity distributions that directly reflect the actual molecular distributions. As shown in Fig. 3B, the label-free biomedical imaging patterns of three 
Raman bands-3400 $\mathrm{cm}^{-1}$ (water), $2490 \mathrm{~cm}^{-1}$ (lipid), and $1650 \mathrm{~cm}^{-1}$ (protein) - were shown for two different water concentrations, low and high, in the same cell. The $2940 \mathrm{~cm}^{-1}$ band was assigned to the stretching modes of the $\mathrm{CH}$ bonds of lipids, the primary components of biological membranes, and $1650 \mathrm{~cm}^{-1}$ was dominated by secondary protein structures (8).

According to the information contained in these label-free and high-contrast images, water distribution was nearly uniform throughout the entire cytoplasm and nucleus, however there were some hydrophobic sites. Moreover, the lipids and proteins appeared to localize at the hydrophobic sites. Although the sites are yet to be identified, in our opinion, they would be assigned to lipid droplets because of numbers, sizes and locations in the cell. Lipid droplets which occur in various numbers and sizes in the cytoplasm of HL-60 cells, form the main lipid store in eukaryotic cells, and play a crucial role in regulating cellular lipid levels (1). Neutral lipids are deposited between the two monolayers of the endoplasmic reticulum membrane, and there they aggregate into a three-dimensional droplet, which buds and pinches off from the endoplasmic reticulum membrane. If the hydrophobic sites are protein-rich and lipid-rich organelles, they can be assigned to mitochondria. This is because mitochondrial matrix contains a highly concentrated mixture of hundreds of enzymes, including those required for the oxidation of pyruvate and fatty acids for the citric acid cycle (1). Further investigation of these hydrophobic sites is presently difficult due to biospectroscopic technical limitations.

The images also showed that the molecular distributions of lipids and proteins appeared clearer at low water concentration than at high water concentration. These distributions were completely random and disordered at high concentrations, probably due to the movement of "surplus" water. The random distributions are considered to be the result of a breakdown in intracellular water homeostasis, because water is central to the highly-organized molecular intracellular network of weak non-covalent connections. The surplus water can confound intracellular homeostasis, disrupt various cellular functions, and offer a possible promoting mechanism for acquiring abnormal cellular functions.

Although further investigation of the detailed molecular role of intracellular hydrodynamics in regulating cellular functions is needed, this biospectroscopic investigation of intracellular water concentration has great potential in determining cell specificity for future use in medicine. Given the fundamental participation of intracellular hydrodynamics in determining cell specificity, this method has significant potential in developing label-free noninvasive therapeutic techniques for targeting abnormal cells. For example, if this biospectroscopic analysis can non-invasively sort the patient's blood into normal constituents and abnormal cells such as cancer cells, virally infected cells, etc., it must be very useful in assessing hematological therapies.

In conclusion, our results demonstrated that intracellular hydrodynamics is a type of biomarker indicating cell functions and supporting the clinical perspective that the degree of malignancy increases with the extent of cell hydration. We also proposed that a combination of confocal Raman microspectroscopy and imaging is a highly useful approach for detecting the intracellular hydrodynamics and monitoring biochemical processes associated with the properties of water in intact cells. Given the importance of intracellular water homeostasis, this biospectroscopic method has the great potential to provide significant insights for molecular cell biology, and also shows tremendous promise in medicine.

\section{Acknowledgement}

We thank Drs. T. Awatani and N. Kojima (Nissan ARC, Japan) for helpful discussions and technical assistance; Dr. M. Yamada (Yokohama City University, Japan) for providing us with HL-60 cells; Dr. T. Takamatsu (Kyoto Prefectural University of Medicine, Japan) for helpful discussions; and Ms. S. Kanaya (Yokohama City University, Japan) for secretarial assistance. This work was supported by Yokohama Foundation for the Advancement of Medical Science (to HF) and by ICHIJU Industrial Science and Technology Promotion Foundation (to HF).

\section{REFERENCES}

1. Alberts B, Johnson A, Lewis J, Raff M, Roberts K and Walter P (2008) Molecular Biology of the Cell, fifth edition. Garland Science, New York.

2. Ball P (2008) Water as an active constituent in cell biology. Chem Rev 108, 74-108.

3. Chaplin M (2006) Do we underestimate the importance of water in cell biology? Nat Rev Mol Cell Biol 7, 861-866.

4. Freudiger CW, Min W, Saar BG, Lu S, Holtom GR, He C, Tsai JC, Kang JX and Xie XS (2008) Label-free biomedical imaging with high-sensitivity by stimulated Raman scattering microscopy. Science 322, 1857-1861.

5. Gallagher R, Collins S, Trujillo J, McCredie K, Ahearn M, Tsai S, Metzgar R, Aulakh G, Ting R, Ruscetti F and Gallo $\mathrm{R}$ (1979) Characterization of the continuous differentiating 
myeloid cell line (HL-60) from a patient with acute promyelocytic leukemia. Blood 54, 713-733.

6. Mclntyre GI (2006) Cell hydration as the primary factor in carcinogenesis: a unifying concept. Med Hypotheses 66, 518526.

7. Ogawa M, Harada Y, Yamaoka Y, Fujita K, Yaku H and Takamatsu T (2009) Label-free biochemical imaging of heart tissue with high-speed spontaneous Raman microscopy. Biochem Biophys Res Commun 382, 370-374.

8. Pelletier MJ (1999) Analytical Applications of Raman Spectroscopy. Blackwell Science, Oxford.
9. Potma EO, de Boeij WP, van Haastert PJM and Wiersma DA (2001) Real-time visualization of intracellular hydrodynamics in single living cells. Proc Natl Acad Sci USA 98, 15771582 .

10. Puppels GF, de Mul FFM, Otto C, Gerve J, Robert-Nicoud M, Arndt-Jovin DJ and Jovin TM (1990) Studying single living cells and chromosomes by confocal Raman microspectroscopy. Nature 347, 301-303.

11. Swain RJ and Stevens MM (2007) Raman microspectroscopy for non-invasive biochemical analysis of single cells. Biochem Soc Trans 35, 544-549. 